\title{
THE DETECTION OF JUNCTION FEATURES IN IMAGES
}

\author{
Robert Laganière and Rimon Elias
}

\author{
VIVA Research Lab \\ School of Information Technology and Engineering \\ University of Ottawa \\ Ottawa, Ontario, Canada, K1N 6N5 \\ laganier@site.uottawa.ca
}

\begin{abstract}
This paper presents a new junction detection operator that defines junctions as points where linear ridges in the gradient domain intersect. The radial lines that compose the junction are therefore identified by searching, in a circular neighborhood, for directional maxima of the intensity gradient. The proposed algorithm operates on two binary edge maps, the computational complexity of the detection process is then considerably reduced.
\end{abstract}

\section{INTRODUCTION}

In image analysis, many important algorithms rely on the automatic detection of interest points. These are distinctive features of an image carrying meaningful information. In the past, several works have proposed various operators that can detect interest points. Most of the time, these operators look for prominent features that correspond to highcurvature boundaries in the image. For this reason, the detected features are often referred to as corners even if, in reality, these operators are sensitive to a wider variety of intensity patterns.

An alternative way of defining interest points is to describe them as image points where several edges meet. Interest points that comply with this definition could be called junctions. The number of converging edges and their respective orientations constitute then the signature characterizing the junction. In fact, the existence of such characterization constitutes probably the way of distinguishing junction detectors from corner detectors. Indeed, most interest point detectors only output the strength of their detected corner. Once a threshold is applied to these values, the subsequent processing stages are left with a binary image carrying only information about feature location. In contrast, a junction detector would extract the relevant attributes of the detected feature, thus subsuming a more informative model of the point of interest and of its vicinity.

This paper presents a new junction detection algorithm that can be used as an interest point detection operator. It assumes that the branches of the detected junctions can be approximated as straight lines in the vicinity of the point of convergence. These junction radial lines can then be found from the identification of what we have called circumferential anchor points.

Other methods for characterizing junctions have been proposed in the past. However, their computational complexity generally do not allow them to be used for junction detection and localization. Instead, they would be applied on selected interest points having been previously detected by some efficient corner detector. This is the case of the method proposed in [1] which is based on the successive application of rotated wedge averaging filters around a given keypoint, each of them computing the averaged accumulated intensity along a radius of particular angle. It results a $1 \mathrm{D}$ intensity profile in which the junction edges correspond to maxima in its first derivative. The approach described in [2] detects junctions by fitting a junction model made of a piecewise constant function that partitions a circular template into wedge-shaped regions. Although the method leads to an effective dynamic solution, the required computational effort remains important. The corner detector in [3] is based on a similar approach where junctions are detected by minimizing the sum of squared differences between a parametric model and the observed intensity values. The work presented in [4] is also another approach based on the use of a model. All these works proceed by examining the intensities inside a defined circular template; consequently, they are usually referred to as region-based approaches. An alternative strategy consists in considering the edge image in order to explicitly identify the junction branches; the methods using this approach are called edgebased approaches. This categorization is well described in [5] where two methods for junction detection are proposed and compared. The first one, that is region-based, uses a greedy algorithm to identify intervals of homogeneous intensity in the junction's 1D intensity profile. The second, edge-based, approach proceeds by accumulating, 
along radius corresponding to each considered direction, evidence of the presence of an edge corresponding to a junction wedge limit. In [6], a junction detector is proposed that makes use of the local properties of the gradient magnitude near junction points in order to reconnect broken edges. In [7], junctions are detected by measuring the rate of change of the orientation vector along lines within a given neighborhood. The detector in [8] uses a two-pass Hough transform to identify points of intersection in an edge map.

The rest of the paper is organized as follows. The next section discusses the problem of junction detection. Section 3 describes the proposed JUDOCA algorithm. Some experimental results are presented in Section 4. Finally, Section 5 is a conclusion.

\section{DETECTING JUNCTIONS}

Junctions in images occur when several uniform regions join at one prominent point, i.e. the point of junction, where the boundaries of the adjacent regions meet. When a junction is produced by only two regions, this definition assumes a high curvature boundary where the point of junction corresponds to the point of maximal curvature (in this case, we have a simple 'corner').

In the gradient domain, points of junction correspond to points where ridges of the image converge. This suggests that junction detection can be accomplished from gradient information. In order to facilitate the detection of junctions, the class of junctions to be detected can be restricted to those where the joining edges can roughly be approximated by straight lines in the vicinity of the point of junctions. The detector proposed in this paper defines the vicinity of a putative point of junction as a circular neighborhood of radius $\rho$ and centered at the point of junction. The radial lines that form the junction must therefore lie on some rays of this disk, as illustrated in Figure 1. The following two properties should hold for such junctions:

1. The value of the gradient of the points of intersection between the circle that delimitates the junction's neighborhood and each of the junction radial lines must correspond to a local maximum in the direction of the gradient (a ridge point).

2. The value of the gradient along the ray on which each junction branch lies must always be greater than a predetermined value (not necessarily on a ridge though, in order to permit some minor deviations from the straight-edge model).

Note that constraints on the orientation of the gradient on each junction radial line could have been added. However, it can be shown that the above two properties somewhat subsume that the gradient orientation will be consistent with the

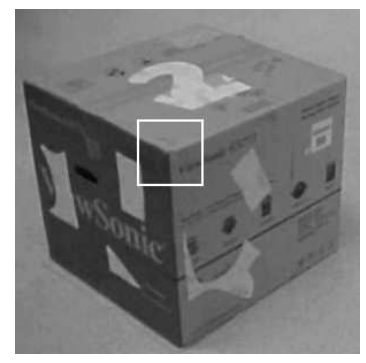

(a)



(b)
Fig. 1. (a) One corner of a box that produces a Y-junction. (b) The junction at $p$ with three circumferential anchors $q_{1}, q_{2}$ and $q_{3}$ (superimposed on the gradient image).

orientation of each junction branches. It follows that there will be a junction at a given image point if at least two rays obeying properties 1 and 2 can be emitted from this point. Also, the strength of such a junction should be proportional to the energy of the gradient along each accepted ray.

Properties 1 and 2 form the basic principles through which junction detection and characterization can be performed. An operator that makes use of these properties is presented in the next section. The underlying algorithm is based on the identification of local directional maxima of the intensity gradient values located on the circumference of a circular neighborhood. When devising this algorithm, one concern was to obtain an operator that can perform junction detection in an efficient manner. For this reason, it operates on binary edge maps. This simplification significantly reduces the computational cost of the method while still detecting junctions that adhere to properties 1 and 2. The proposed algorithm has been named JUDOCA for JUnction Detection from Circumferential Anchor points.

\section{THE JUDOCA ALGORITHM}

To detect junctions, the proposed operator relies on the intensity gradient. This one can be conveniently estimated using Gaussian derivatives filters. The size of the Gaussian filter, which is specified by a variance parameter $\sigma$ also offers the advantage of allowing edge detection at different scales, i.e. only more prominent edges being detected for larger value of $\sigma$. Note, however, that if good localization is required, smaller $\sigma$ value should be used.

The algorithm proceeds as follows:

1. Apply vertical and horizontal Gaussian derivative filters on image $\mathbf{I}$.

2. Compute the gradient magnitude and create two binary images from it: 
(a) The first one, $\mathbf{B}$, is created by imposing a threshold, $t_{B}$, on the gradient image.

(b) The second one, $\mathbf{B}^{+}$, contains the points of $\mathbf{B}$ that are local maxima in the direction of the gradient.

3. For each point $p$ in $\mathbf{B}$, consider a circle of radius $\rho$ centered on this point and obtain the list of candidate points $q_{i}$ in $\mathbf{B}^{+}$that lie on the circumference of this circle (see Figure 1). These so-called circumferential anchor (CA) points are the extremities of potential radial lines for the putative junction.

4. For each CA point $q_{i}$ in the list, consider the set of all points located at a distance less than one pixel to the segment that joins the current CA point to the central point $p$. This set is used to determine if the corresponding putative junction radial line will be accepted (considering the points in $\mathbf{B}$ ) and if yes, what the strength of this junction line would be (considering the points in $\mathbf{B}^{+}$), that is:

(a) To be accepted as a junction line, a continuous path of $\mathbf{B}$ points joining the CA point and the central point $p$ must exist. If not, then reject this radial line and repeat the scanning operation with the next CA point.

(b) The strength, $S_{J}\left(<p, q_{i}>\right)$, of this junction radial line is defined as the sum of the squared distances from the $\mathbf{B}^{+}$points in the currently considered set to the $\left\langle p, q_{i}>\right.$ line segment. This strength is normalized by the length of this segment.

5. If the strength of this junction radial line is smaller than a predetermined threshold, $s_{J}$, then reject this radial line. Otherwise, $<p, q_{i}>$ becomes one of the branch of the putative junction at $p$.

6. If the number of branches found at $p$ is less than 2 , then there is no junction at this location. Otherwise, record the orientation of the accepted junction radial lines and set the strength, $S_{J}(p)$, of this junction as being the minimum across all radial line strengths, i.e.:

$$
S_{J}(p)=\min _{i} S_{j}\left(<p, q_{i}>\right)
$$

In the particular case of 2-junction, an extra step must be undertaken in order to ensure that this junction is not, in fact, a simple line. This can be verified by looking at the angle between the two radial lines. If this one is close to $180^{\circ}$, then the junction should be rejected. In practice, a threshold is set on the maximal and minimal acceptable angle for 2junctions.
Additionally, the strength of the junction can be used to perform a non-maxima suppression postprocessing phase to eliminate clusters of junction that could arise, especially if a permissive threshold is used in Step 5. Different definitions for the junction strength could have been adopted. However, the one given in Step 6 is based on an assertion stating that a junction is as weak as its weakest branch. This definition also implies that a weak $N$-junctions can be transformed into a stronger $(N-1)$-junction.

\section{EXPERIMENTAL RESULTS}

In the JUDOCA algorithm, four parameters control the detection of junctions. The first two, $\sigma$ and $t_{B}$, concern the creation of the two binary edge maps, $\mathbf{B}$ and $\mathbf{B}^{+}$. Their values influence the sensitivity of the detection and determine the scale at which the junction detection is performed. For example, comparing Figure 3(a) to 3(b), it appears that several textural details can be filtered out, if wished, by a proper choice of $\sigma$. Parameter $\sigma$ plays also an important role regarding the noise robustness of the operator. This is illustrated in Figure 2 where the detector has been tested on a synthetic image to which Gaussian noise has been added. By increasing the value of $\sigma$, it has been possible to considerably reduce the impact of noise on the detection of junctions. In other cases, noise effect can be overcome by reducing the edge detection threshold, $t_{B}$.

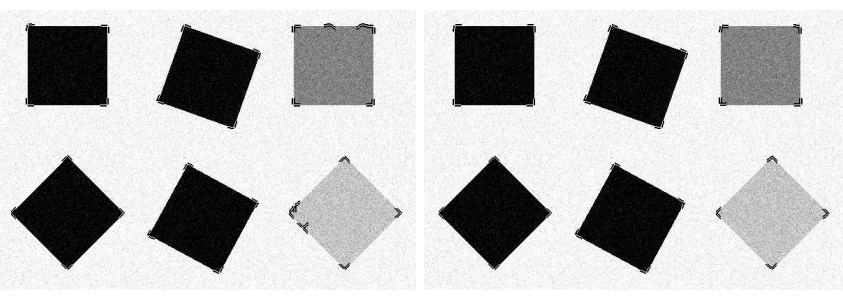

(a)

(b)

Fig. 2. Junction detection on a synthetic test image with Gaussian noise of variance $=400$. (a) Detected junctions with $\sigma=1.0$. (b) Detected junctions with $\sigma=1.5$.

The length, $\rho$, of the radial lines that are scanned during the detection process has an impact on the number of found junctions. Normally longer radial lines are more desirable since they better support the defined straight-edge junction model. By choosing a smaller length, the detector will act as a 'regular' corner detector sensitive to prominent high curvature points. In Figure 3(c), a smaller radial length has been used, compared to Figure 3(a), resulting in an increase in the number of detected junctions (125 detected junctions with $\rho=7$ compared to 79 with $\rho=10$ ). We found that values between 8 and 20 usually give good results.

The accuracy of the junction orientation is also better 
with larger value of $\rho$. To demonstrate this fact, we took different pictures of a triangular shape (28 in total). Knowing that, no matter what the point of view is, the sum of the three angles should always be $180^{\circ}$, we computed for each of these images, the sum of the junction angles as detected by JUDOCA. We repeated this experiment for different values of $\rho$. As expected, the mean value of this sum approaches $180^{\circ}$ as $\rho$ increases (from $202^{\circ}$ to $187^{\circ}$ for $\rho$ between 8 and 20). Also the variance of this sum decreases with increasing $\rho$. This shows that not only do longer radial lines increase the accuracy of the angles but also make these measurements more reliable.

Finally, the threshold $s_{J}$ defines the degree of adherence to the straight edge assumption. Indeed, because of noise, shading and contrast variation, the edge contours in $\mathbf{B}^{+}$will not be perfectly straight. Consequently, a level of tolerance must be defined for the acceptance of a junction. If one wants to perform a more selective junction detection, the value of this threshold can be made higher, at the price of missing some good junctions. Typically $s_{J}$ varies between $50 \%$ and $90 \%$. This percentage roughly represents the minimal acceptable portion of the radial line having a $\mathbf{B}^{+}$point close to it. Obviously, according to Equation (1), the strength of any accepted junction will always be higher than this value.

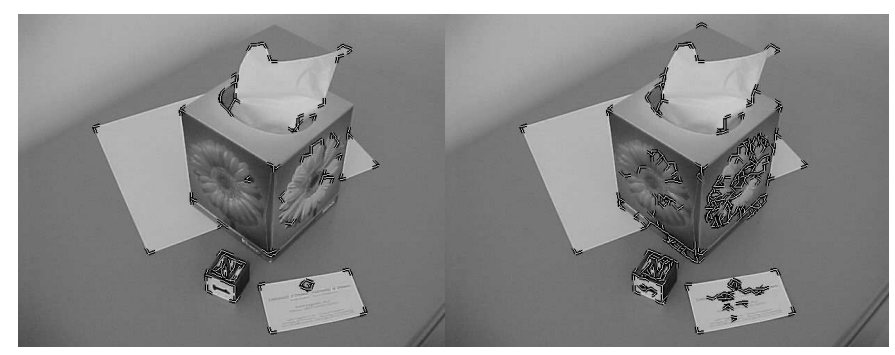

(a)

(b)

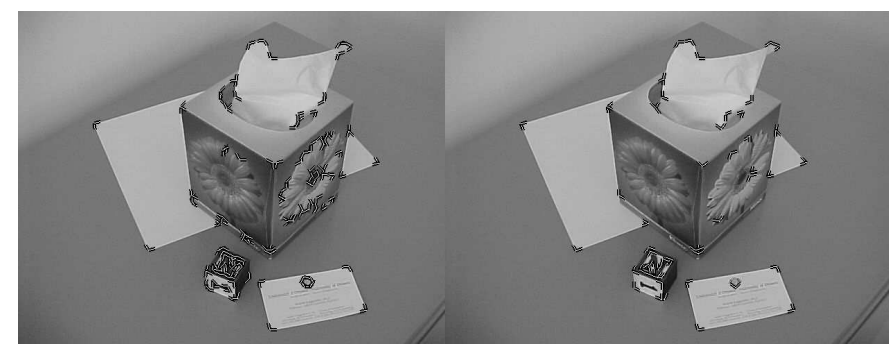

(c)

(d)

\section{CONCLUSION}

The JUDOCA algorithm presented in this paper is a junction detection operator that detects junctions and characterizes them in terms of junctions strength, number of branches and junctions orientation. Because of its efficiency, this operator can be used as an interest point detector, providing rich information to subsequent steps, such as matching. Comparative tests with other methods still have to be made, however Cazorla [5] reports an execution time of 7s and 4s for his two methods to detect 100 junctions on $640 \times 480 \mathrm{im}$ ages using a PII 266MHz. With JUDOCA, we measured an excution time of $1.1 \mathrm{~s}$ to compute the two binary edge maps and $0.5 \mathrm{~s}, 1.49 \mathrm{~s}, 2.4 \mathrm{~s}$ to detect 140, 236, 409 junctions on different images using a similar machine. An implementation of the JUDOCA algorithm has been made available to interested researchers through our Web site. ${ }^{1}$

\section{REFERENCES}

[1] G. Sommer W. Yu, K. Danilidis, "Rotated wedge averaging method for junction characterization," in CVPR, 1998, pp. 390-395.

[2] R. Hummel L. Parida, D. Geiger, "Junctions: Detection, classification, and reconstruction," IEEE PAMI, vol. 20, pp. 687-698, 1998.

[3] K. Rohr, "Modelling of intensity variations," Image Vision Computing, vol. 10, pp. 66-76, 1992.

[4] M.A. Ruzon and C. Tomasi, "Edge, junction and corner detection using color distributions," IEEE PAMI, vol. 23, no. 11, pp. 1281-1295, November 2001.

[5] M.A. Cazorla and F. Escolano, "Two bayesian methods for junction classification," IEEE trans. on Image Processing, vol. 12, no. 3, pp. 317-327, March 2003.

[6] D.J. Beymer, "Finding junctions using the image gradient," in CVPR, 1991, pp. 720-721.

[7] D. Ziou F. Deschenes, "Detection of line junctions in gray-level images," in ICPR, 2000, vol. 3, pp. 754-757.

[8] W. A. Barrett and Kevin D. Petersen, "Houghing the hough: Detection of corners, junctions and line intersections," in CVPR, 2001, vol. II, pp. 302-309.

Fig. 3. Junction detection on a simple image using JUDOCA with (a) $\sigma=1.8, \rho=10$ and $s_{J}=0.6$. (b) $\sigma=1.0$, $\rho=10$ and $s_{J}=0.6$. (c) $\sigma=1.8, \rho=7$ and $s_{J}=0.6$. (d) $\sigma=1.8, \rho=10$ and $s_{J}=0.7$.

\footnotetext{
${ }^{1}$ www.site.uottawa.ca/school/research/viva/projects/judoca
} 